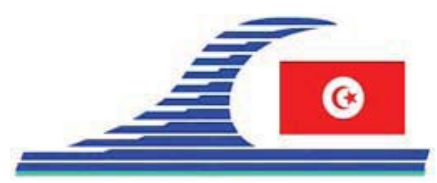

Conférence Méditerranéenne Côtière et Maritime EDITION 1, HAMMAMET, TUNISIE (2009)

Coastal and Maritime Mediterranean Conference

Disponible en ligne - http://www.paralia.fr-Available online

\title{
Réhabilitation de l'appontement de Crozet par chemisage PEHD - Terres Australes et Antarctiques Françaises (TAAF)
}

\author{
Hervé BARREDA ${ }^{1}$, Laurent BESNARD ${ }^{2}$, \\ Philippe DELORME ${ }^{3}$, Henri GOUGE ${ }^{4}$
}

1 CETMEF Agence d'Aix en Provence, 2 boulevard du Président Kennedy CS90385, 13097 Aix en Provence cedex 2, France.

herve.barreda@developpement-durable.gouv.fr

2. TAAF, Rue Gabriel Dejean, BP 400, 97410 Saint Pierre de La Réunion, France. laurent.besnard@taaf.fr

3. Conseil Général de l'Ariège, 5 Rue Cap de la Ville, 09000 Foix, France. philibertdelorme@free.fr

4. DDE des Bouches du Rhône, 1 rue Vincent Auriol, BP 60781, 13625 Aix en Provence cedex 01, France.

henri.gouge@developpement-durable.gouv.fr

\section{Résumé :}

L'article expose les actions de surveillance et de maintenance réalisées sur l'appontement de Crozet, soit : l'expertise dans un environnement hostile et isolé (situé à $3000 \mathrm{~km}$ de La Réunion et uniquement accessible par voie maritime) et la réhabilitation par chemisage anticorrosion PEHD (Polyéthylène Haute Densité) des nouveaux pieuxtubes métalliques.

La méthode de protection anticorrosion utilisée est applicable aux ouvrages dont les pieux sont fondés, en zone de déferlement de la houle, par fond meuble (plage de sable et/ou de graviers). Il s'agit d'une méthode préventive ou curative innovante et originale : simple au niveau de la mise en œuvre, efficace au niveau anticorrosion, peu onéreuse, n'ayant pas d'impact sur l'environnement.

\section{Mots-clés :}

Houle - Appontement - Pieu-tube - Protection anti-corrosion - Corrosion Réhabilitation - Chemisage PEHD (Polyéthylène Haute Densité) - Système duplex

\section{Description et historique de l'ouvrage}

1974 : Construction de l'appontement : 22,10 m de long sur 6,00 m de large et 0,45 m d'épaisseur du tablier, en béton armé (BA). Le tablier est supporté par 18 pieux. Depuis l'enracinement l'appontement comporte 7 files de pieux qui se décomposent comme suit : files 1 à 5,2 pieux/file, puis files 6 et 7, 4 pieux/files.

DOI: $10.5150 / \mathrm{cmcm} .2009 .001$ 
2004 (août) : L'expertise, réalisée par le CETMEF, révèle que tous les pieux sont très corrodés. Ces atteintes sont de nature à compromettre la stabilité de l'ouvrage. Le CETMEF préconise une solution de réhabilitation anticorrosive.

2005/2006 (été austral) : Réalisation des travaux par une entreprise réunionnaise SGTPS, titulaire du marché, notifié par les TAAF.

\section{L'expertise}

Récapitulatif des opérations menées in situ :

- inspection visuelle de l'ouvrage et relevé des désordres ;

- mesures par ultrasons des épaisseurs résiduelles des pieux ;

- mesures des potentiels électrochimiques des pieux (situation électrochimique);

- bathymétrie, composition des fonds et lançage : vérification de l'épaisseur de sable et de la profondeur du refus ;

- prélèvements de sol pour analyse des matériaux (granulométrie) et d'eau (résistivité).

\section{Inspection visuelle de l'ouvrage}

Après 30 années de service (1974 à 2004) le tablier a bien résisté aux actions de la houle. Les pieux très affectés par la corrosion présentent, hors d'eau, un foisonnement généralisé. Dans la zone de déferlement le métal apparaît blanc brillant (corrosion/abrasion). Les 2 pieux de la file 5 (figure non fournie) sont complètement détruits à la base, sur $0,40 \mathrm{~m}$ de haut.

\section{Lançages et reconnaissance du fond}

Le lançage le plus profond (point d'origine du profil Sud) est de 1,00 m, les autres ont entre 0,40 à $0,60 \mathrm{~m}$ de profondeur. On observe une prédominance de sable noir cru d'origine basaltique sans sédiment meuble et le refus n'est pas franc. Le fond, légèrement en pente d'environ (7 à 20\%), est essentiellement constitué de sable noir.

\section{Hauteurs des pieux}

Les hauteurs montrent que le profil a une tendance à l'engraissement en haut de plage et à un équilibre en bas, du côté mer. Pas de surcreusement significatif mis en évidence.

\section{6. Épaisseurs résiduelles et potentiels électrochimiques}

Sur 18 pieux, 3 ne participent plus au fonctionnement de la structure. Les pieux, affectés d'une vitesse de corrosion importante (corrosion/abrasion), ont perdu de 75 à $100 \%$ d'épaisseur initiale. A noter que les pieux sont bétonnés et armés.

Les potentiels confirment que les pieux ne sont pas perturbés par des courants vagabonds ou d'anomalies électrochimiques. Ils sont conformes au potentiel d'abandon de l'acier en eau de mer, qui est compris entre -550 à -690 mV Ag/AgCl/eau de mer. 


\section{Conclusion et préconisation de réhabilitation}

Tous les pieux sont très affectés par la corrosion. Les désordres affectant les pieux des files 5,6 et 7 sont de nature à compromettre la stabilité de l'ouvrage. Il conviendra de réhabiliter l'appontement par le remplacement de tous les pieux. Ils seront chemisés avec du PEHD (LEMAIRE, 1988 ; BARREDA et al., 2002 ; LABEL IVOR, 2002 ; ARAGON et al. 2006) pour les préserver de la corrosion/abrasion. En configuration de déferlement, la solution protection cathodique par anodes sacrificielles très difficile à mettre en œuvre pour une efficacité incertaine (BARREDA \& LAZZERI, 1995), n'a pas été retenue.

\section{La réhabilitation et les 7 contraintes}

L'intérêt technique tient dans l'originalité de la méthode utilisée : forage du tablier au $\phi$ des nouveaux pieux, battage, découpage du tablier en BA, recépage, chemisage anticorrosion en PEHD, injection d'un mortier de calage dans l'espace annulaire, bétonnage du pieu et du chevêtre puis enlèvement des anciens pieux. Les travaux ont duré, environ, 4 mois in situ (de décembre 2005 à mars 2006).

Ils ont, également, intégré les 7 contraintes suivantes : climatique, éloignement, temps, sécurité, intempérie, psychologique et environnementale.

\section{Contrôle du béton}

Des essais de convenance de béton ont, préalablement, été réalisés en octobre 2005 à la Plaine des Cafres de La Réunion considérée comme étant le lieu se rapprochant le plus des conditions météo de Crozet. Ces essais portant sur trois formulations, ont permis de confirmer : la mise en œuvre et la résistance en compression du béton ( $>35 \mathrm{MPa}$ à 28 jours). Pendant la phase bétonnage à Crozet : confection d'éprouvettes $(\phi 16 \times 32 \mathrm{~cm})$, transport jusqu'à La Réunion puis écrasements en laboratoire. Les valeurs ont révélé des résistances en compression plus élevées que les valeurs attendues ce qui répond aux exigences du fascicule 65A « Exécution des ouvrages de génie civil en béton ».

\section{Mode opératoire de mise en oeuvre}

Réhabilitation de la sous face du tablier en BA :

- piquage et nettoyage des zones altérées (aciers corrodés et épaufrures );

- passivation des aciers apparents (Sika Monotop 610AC) ;

- ragréage et surfaçage avec une résine époxydique (Sika Monotop 630SR).

Tous les 18 pieux atteints, ont été remplacés par 14 nouveaux comme suit :

- carottage du tablier au $\phi 410 \mathrm{~mm}$ au droit d'un nouveau pieu ;

- battage de nouveaux pieux $\phi 406 \mathrm{~mm}$ épais $12.7 \mathrm{~mm}$;

- découpage du tablier $(1.30 \times 1.00 \mathrm{~m})$ au niveau de chaque nouveau pieu ;

- recépage et curage des nouveaux pieux ;

- forage des scellements horizontaux des aciers des chevêtres dans le tablier; 
- mise en place du PEHD et battage d'environ 0,50 m dans le sable ;

- ferraillage des nouveaux pieux ;

- remplissage de l'espace annulaire pieu/PEHD avec un mortier de calage à retrait compensé haute performance. La protection contre la corrosion/abrasion des pieux est assurée par du PEHD ( $\phi 630 \mathrm{~mm}$, épaisseur $30 \mathrm{~mm})$;

- scellements des aciers HA du chevêtre identiques à l'existant dans le tablier ;

- mise en place du coffrage puis bétonnage du nouveau pieu et du chevêtre ;

- suppression des $1 / 2$ coquilles, découpage et enlèvement des 18 anciens pieux.

Le montant de la réhabilitation s'élève à $494000 € \mathrm{HT}$.

\section{Conclusion et perspectives}

La méthode de protection des pieux métalliques utilisant le PHED est innovante, originale, simple au niveau de la mise en œuvre et efficace au niveau anticorrosion. Elle peut être utilisée indifféremment : soit préventivement sur un ouvrage neuf, soit curativement sur un ouvrage en service. Elle présente aussi l'intérêt d'être peu onéreuse, peu consommatrice en matériau et en matériel et a peu d'impact sur l'environnement.

\section{Références bibliographiques}

ARAGON E., BARREDA H., EYRAUD M., PELLON L., VACANDIO F., VERRIER N. (2006). Élaboration d'une méthode d'évaluation des performances de revêtements époxydiques applicables en immersion. $4^{\text {éme }}$ colloque international d'Aix en Provence sur la Protection Cathodique et Revêtement Associé (juin) CEFRACOR Paris.

BARREDA H., LAZZERI A. (1995). Protection cathodique des palplanches en eau de mer : dimensionnement des anodes sacrificielles. Note technique du bulletin de liaison des Ponts \& Chaussées (janvier) n 195, LCPC Paris.

BARREDA H., HUBERT C., FARDELLA C. (2002). Systèmes de protection anticorrosion duplex de structures métalliques à la mer. $2^{\text {iéme }}$ colloque international d'Aix-en-Provence sur la Protection Cathodique et Revêtement Associé (juin) CEFRACOR Paris, pp 181-193.

LABEL IVOR (2002). Procédé anticorrosion duplex par chemisage et protection cathodique de pieux métalliques. $\mathrm{n}^{\circ}$ 02.4, DRAST/Mission Génie Civil/Comité Innovation Validées sur Ouvrages de Référence - Paris la Défense.

LEMAIRE F. (1988). La protection contre la corrosion des structures métalliques immergées. Service Technique Central PMVN, Notice STC-QG, ${ }^{\circ}$ 88-2, Compiègne. 